\title{
Mathematical work of Franciszek Hugon Szafraniec and its impacts
}

\author{
Raúl E. Curto ${ }^{1} \cdot$ Jean-Pierre Gazeau ${ }^{2} \cdot$ Andrzej Horzela $^{3}$ • \\ Mohammad Sal Moslehian $^{4} \cdot$ Mihai Putinar $^{5,6} \cdot$ Konrad Schmüdgen $^{7}$. \\ Henk de Snoo $^{8} \cdot$ Jan Stochel ${ }^{9}$
}

Received: 15 May 2020 / Accepted: 19 May 2020/Published online: 8 June 2020

(C) The Author(s) 2020

\begin{abstract}
In this essay, we present an overview of some important mathematical works of Professor Franciszek Hugon Szafraniec and a survey of his achievements and influence.
\end{abstract}

Keywords Szafraniec $\cdot$ Mathematical work · Biography

Mathematics Subject Classification 01A60 - 01A61 · 46-03 - 47-03

\section{Biography}

Professor Franciszek Hugon Szafraniec's mathematical career began in 1957 when he left his homeland Upper Silesia for Kraków to enter the Jagiellonian University. At that time he was 17 years old and, surprisingly, mathematics was his last-minute choice. However random this decision may have been, it was a fortunate one: he succeeded in achieving all the academic degrees up to the scientific title of professor in 1980. It turned out his choice to join the university shaped the Kraków mathematical community.

Communicated by Qingxiang Xu.

Jan Stochel

Jan.Stochel@im.uj.edu.pl

Extended author information available on the last page of the article 


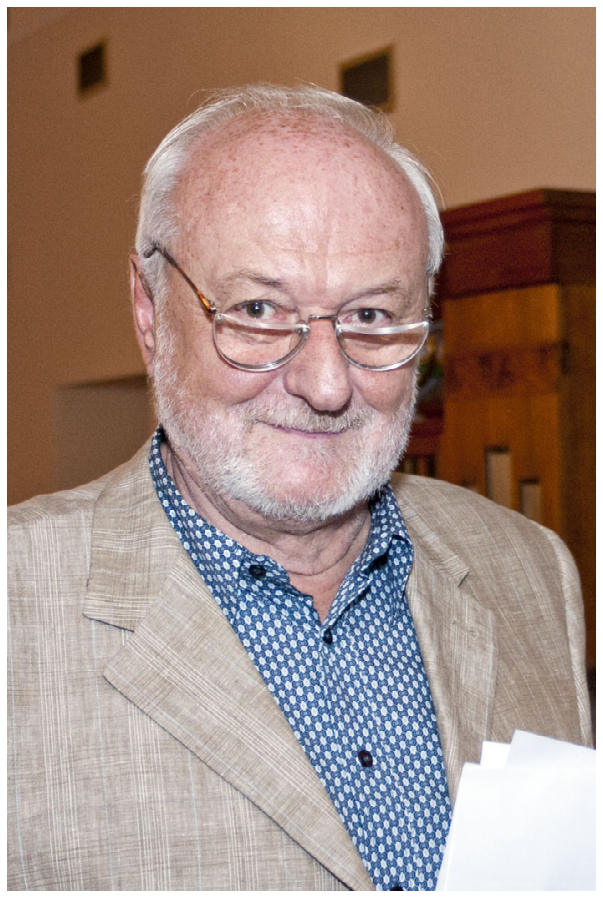

Professor Franciszek H. Szafraniec

Kraków beyond Warsaw and Lwów belonged to the famous Polish School of Mathematics in the prewar period. Kraków was a well known centre of Analysis therefore the School of Differential Equations fitted with it. The main person of the school was Tadeusz Ważewski, who brought from the Paris school topological methods into the subject. Szafraniec was the last PhD student of Ważewski benefiting from his ability to gather brilliant people around himself, directing them to interesting questions and, on the other hand encouraging to break mathematical boundaries. On this ground different members of Ważewski's group spread over diverse areas of mathematics and so did Szafraniec. In 1968 he got converted to Operator Theory by Włodzimierz Mlak and soon after the passion which both of them had for this branch of mathematics was shared by their students and passed on to the next generations of mathematicians. This way Kraków became a vital centre of modern operator theory. The co-workers and former students of Szafraniec are present in all major Kraków universities.

Thematic diversity along with his in-depth insight at mathematical issues is Szafraniec's hallmark. His scientific contribution covers differential equations at first, then followed by a sudden turn into functional analysis and operator theory, and then many related topics including moment problems, orthogonal polynomials, quantum physics, operators in Krein spaces and linear relations. His publication output includes around 140 papers most of which appeared in reputable journals. Szafraniec's activity in the mathematical world together with his ability to cooperate bears fruits in many co-authored publications which encompasses numerous 
papers written together with younger generation of mathematicians, and, as of late, physicists of all ages.

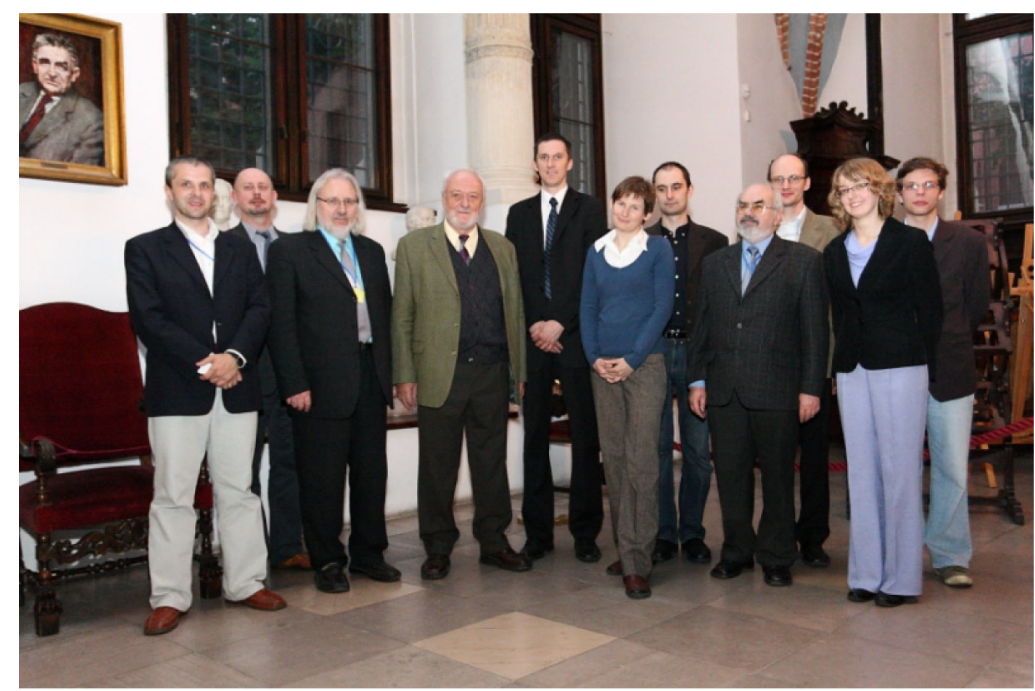

Professor Szafraniec among members of Functional Analysis Group

Until now Szafraniec has been an active participant of many conferences organised all over the world. His talks often go beyond a mere presentation of results, being in fact small performances interspersed with witty insertions. No wonder they gain much attention and their author is often invited to deliver speeches on many occasions, which also form the opportunity for the professor to promote Kraków as a historic city which happens to thrive as a vivid scientific centre. Not only does Szafraniec attend conferences abroad, he also has been organising mathematical meetings in Kraków and in other cities (like Leiden in 2009 and 2013). The conference Functions and Operators (Kraków 2010) was organised on his 70th birthday while the conference 90 years of the reproducing kernel property (Kraków 2000) was a celebration of his 60th birthday. It is worth noting that the reproducing kernel property in question was formulated by Stanisław Zaremba [72] who can be called Szafraniec's scientific grandfather, as an advisor of Ważewski. This motivated Szafraniec to write a unique book devoted to reproducing kernel Hilbert spaces [65].

We refer the reader to [8] for earlier biography of Szafraniec.

\section{The quest for unbounded subnormality}

We are all normal, or at least we believe so. And we know pretty well our own limits, that is we are bounded. Linear operators acting on a Hilbert space are not so fortunate: they can deviate from normality quite a bit, being seminormal, subnormal, hyponormal, paranormal, quasinormal, not to mention that they can be unbounded. 
The dream of all spectral analysts is to deal with an operator displaying a rich spectral decomposition behavior, as close to normality as possible. Even for finite matrices this is too much to ask, not to speak about linear transformations of an infinite dimensional Hilbert space. Identities such as $T=T^{*}$, or $T T^{*}=T^{*} T=I$, or $T T^{*}=T^{*} T$ assure the optimal spectral decomposition behavior a bounded linear operator can possess. However, these ideal and simple to verify algebraic equations are not in general met by multipliers on Hilbert spaces of analytic functions or by linear differential operators. The first class is populated by subnormal operators, that is restrictions of normal operators to an invariant subspace, while the second one is asking for relaxing the boundedness condition, typically imposing a dense domain and a closed graph. Challenges and pitfalls abound in both situations. Ample studies covering half a century led to a good understanding of the nature of a bounded subnormal operator [12]. Even more can be said about von Neumann's groundbreaking spectral theory of unbounded self-adjoint operators, later generalized to other classes of linear transformations, see for instance [3].

It is the merit of Szafraniec and Stochel to have pursued with obstinacy the study of unbounded subnormal operators. They removed with high skill and in style many stumbling blocks arising on the path, even from the very beginning, starting with the definition of this class of operators. Their works excel in rigor and clarity of exposition and they are rooted in current problems of Mathematical Analysis or Quantum Physics. Their articles on this very topics are quite ramified. Without aiming at completeness we offer in the next couple of pages a glimpse into a single aspect of the Szafraniec-Stochel theory of unbounded subnormal operators. The reference list below gives a better picture of the magnitude of the strive.

Let $T$ be a linear operator with dense domain $\mathcal{D}(T)$ of a Hilbert space $\mathscr{H}$. Then the adjoint operator $T^{*}$ is defined. The operator $T$ is called formally normal if $\mathcal{D}(T) \subseteq \mathcal{D}\left(T^{*}\right)$ and $\|T \varphi\|=\left\|T^{*} \varphi\right\|$ for all vectors $\varphi \in \mathcal{D}(T)$ and $T$ is said to be normal if $T$ is formally normal and $\mathcal{D}(T)=\mathcal{D}\left(T^{*}\right)$. In the literature, the following characterization is often taken as a definition of normality: A dense defined operator is normal if and only if $T$ is closed and $T T^{*}=T^{*} T$. The distinction between formally normal operators and normal operators is only important for unbounded operators, because each formally normal operator defined on the whole Hilbert space is obviously bounded and normal.

Normal operators stand aside. First of all, the spectral theorem holds, that is, for each normal operator $T$ there exists a unique spectral measure $E_{T}$ on the Borel $\sigma$ algebra of $\mathbb{C}$ such that $T=\int z E_{T}(z)$. This spectral measure allows one to develop a functional calculus. For each Borel function $f$ on the complex place there exists a unique normal operator $f(T)$ on $\mathbb{C}$ defined by $f(T)=\int f(z) d E_{T}(z)$.

For normal operators there is a natural notion of permutability: we say two normal operators $T_{1}$ and $T_{2}$ acting on the same Hilbert space strongly commute if there spectral measures commute, that is, $E_{T_{1}}(M) E_{T_{2}}(N)=E_{T_{2}}(N) E_{T_{1}}(M)$ for arbitrary Borel sets $M, N$. Further, for each $n$-tuple $T=\left(T_{1}, \ldots, T_{n}\right)$ of pairwise strongly commuting normal operators there exists a unique spectral measure $T$ on $\mathbb{C}^{n}$ such that $T_{k}=\int z_{k} d E_{T}(z), k=1, \ldots, n$. This observation has immediate applications to the multidimensional complex moment problem. 
It is obvious from the definition that self-adjoint operators are normal and densely defined symmetric operators are formally normal. A classical result due to Naimark [37] states that each symmetric operator has an extension to a self-adjoint operator in a possibly larger Hilbert space. In contrast, formally normal operators do not extend in general to normal operators in larger Hilbert spaces. This was first shown by Coddington [10], see [42] for a very simple example.

A densely defined linear operator $T$ on a Hilbert space $\mathscr{H}$ is called subnormal if there exists a normal operator $N$ on a Hilbert space $\mathscr{G}$ which contains $\mathscr{H}$ as a subspace such that $T \subseteq N$. Subnormal operators are formally normal. It is difficult to decide whether or not a formally normal operator is subnormal or normal. The creation operator $A_{+}=\frac{1}{\sqrt{2}}\left(x-\frac{d}{d x}\right)$ of quantum mechanics is subnormal; this fact was nicely elaborated by Szafraniec [67] to a number of operator-theoretic characterizations of the creation operator.

A systematic study of unbounded subnormal operators was begun by Stochel and Szafraniec in the mid eighties in the trilogy of fundamental papers [46-48] and continued since then in a number of research papers, see the reference list below and [68] for a leisure discussion.

The first main problem about unbounded subnormal operators is to decide whether a formally normal operator is subnormal or even normal. This is a difficult problem that has many facets. There is also a natural multivariate version of the problem: It asks when a family, or a commutative *-semigroup, of pairwise commuting formally normal operators has an extension to pairwise strongly commuting normal operators in a possibly larger Hilbert space. One approach is based on the presence of sufficiently many common "well-behaved" vectors. In the most general setting these are quasianalytic vectors, an idea that goes back to the work of Nussbaum [38]. In the first paper [45] of the trilogy this was elaborated in detail and a number of basic results were obtained. Another type of characterizations of subnormality based on positivity conditions is developed in [47]. All these results have natural applications to the (multidimensional) complex moment problem [50] which was both a driving force and a source of important examples for the theory.

A second main problem concerns the relation of subnormal operators to its normal extensions. In particular, existence and properties of minimal extensions are important. In contrast to the bounded case, there are in general different notions of minimality. A normal extension $N$ on a Hilbert space $\mathscr{G}$ of a subnormal operator $T$ on a Hilbert space $\mathscr{H}$ is called minimal of spectral type if $\mathscr{G}$ is the only reducing subspace of $N$ which contains $\mathscr{H}$. The third work [48] of the trilogy is devoted to this area. Two kinds of minimal extensions, those of spectral type and those of cyclic type, are investigated.

\section{Moment problems}

Inverse problems naturally occur in many branches of science and mathematics. An inverse problem entails finding the values of one or more parameters using the values obtained from observed data. A typical example of an inverse problem is the 
inversion of the Radon transform. Here a function (for example of two variables) is deduced from its integrals along all possible lines. This problem is intimately connected with image reconstruction for X-ray computerized tomography.

Moment problems are a special class of inverse problems, and they arise naturally in statistics, spectral analysis, geophysics, image recognition, and economics. While the classical theory of moments dates back to the beginning of the 20th century, the systematic study of truncated moment problems began only a few years ago. In his 1987 seminal paper [32], Landau wrote, "The moment problem is a classical question in analysis, remarkable not only for its own elegance, but also for the extraordinary range of subjects, theoretical and applied, which it has illuminated".

Szafraniec has made numerous contributions to the theory of complex moment problems, including the outstanding research reported in [49, 50, 57], and more recently the work presented in $[9,70,71]$. Some of these results include a novel approach to the complex moment problem, that is, linking positive linear functionals $L$ acting on polynomials $p$ in $z$ and $\bar{z}$ with $d$-tuples $N \equiv\left(N_{1}, \cdots, N_{d}\right)$ of multiplication operators on the Hilbert space $L^{2}(\mu)$, where $\mu$ is a Radon measure on $\mathbb{C}^{d}$. This is done using the functional calculus for normal $d$-tuples of operators, via the cyclic vector $1 \in L^{2}(\mu)$, as follows:

$$
L(p):=\left\langle p\left(N, N^{*}\right) 1,1\right\rangle=\int_{\mathbb{C}^{d}} p(z, \bar{z}) d \mu(z) .
$$

This approach leads to a fruitful interplay between multivariable operator theory, the theory of positive linear functionals on the space of polynomials, and the theory of complex moment problems. Together with [44], it represents a predecessor of the unprecedented connections, beginning in the early 1990's, among real algebraic geometry, optimization theory, the theory of quadratures in numerical analysis, the theory of moments (full and truncated), the mathematics of finance, and the theory of realizability of point processes.

As a simple example of the results obtained by Szafraniec and his collaborators, we recall that the solubility of the moment problem in two variables cannot be characterized in terms of the positivity of the associated moment sequence. This is a consequence of the existence of nonnegative polynomials in two variables that do not admit a representation as a sum of squares of polynomials. In [49], the authors describe a series of additional conditions which allow a positive definite sequence to become a moment sequence, with a representing measure. These conditions have to do with the support of the representing measure, which must belong to a suitable class of algebraic curves. Along the way, the authors prove a boundedness criterion for formally normal operators in Hilbert spaces. In this way, results about moment sequences can be derived from criteria for essential normality for unbounded Hilbert space operators. The work makes contact with a pioneering result of Schmüdgen [43], which created a new bridge between operator theory and real algebraic geometry.

In another trailblazing research accomplishment, in joint work with Stochel, Szafraniec discovered a polar decomposition approach to the moment problem. 
Consider a double-indexed sequence $\gamma \equiv\left\{\gamma_{m, n}\right\}$ of complex numbers, where the indices $m$ and $n$ run over the integer lattice points of the nonnegative quarter plane; that is, whenever $m, n \geq 0$. Solving the moment problem entails, in this case, finding a positive Borel measure $\mu$ on $\mathbb{C}$ such that $\gamma_{m, n}=\int z^{m} z^{n} d \mu(z)($ all $m, n \geq 0)$. It is well known that the existence of a representing measure for $\gamma$ implies that $\gamma$ is positive definite, that is, the associated moment matrix must be positive semidefinite. It is also known that this condition is not sufficient for the solubility of the complex moment problem. Now suppose that we ask $\gamma$ to admit a positive definite extension $\Gamma$ to the integer lattice points of the northeast half-plane determined by the diagonal $m+n=0$; that is, $\Gamma$ must be positive definite for all pairs $(m, n)$ such that $m+n \geq 0$. In [50], the authors proved that $\gamma$ has a representing measure if and only if the above mentioned extension $\Gamma$ exists.

This superb result was highlighted in a Featured Mathematical Review, alongside another superb result obtained by Putinar and Vasilescu [40]. The two articles represented outstanding additions to our existing knowledge, in terms of providing new criteria for existence and uniqueness of representing measures, and for localization of the support of such measures. They introduced original ideas, methods and techniques that had a lasting impact on subsequent developments of the theory. Both articles appealed to the notion of extendability, in different but compatible directions, and consonant with the main approach to truncated moment problems that was being developed at the time. The key ingredient needed was the idea of building a new moment problem, essentially equivalent to (and extending) the original one, but in a higher-dimensional setting, where positivity alone provides the necessary and sufficient condition, just as in the single-variable case. The work in [50] was followed by a paper on determinacy and extendability [9]. More recently, Szafraniec has made substantial contributions to the study of the complex moment problem of Dirichlet type [71], and to the Sobolev moment problem [70].

Szafraniec's ideas are often brilliant, and address fundamental problems; solutions provided indicate a profound understanding of the intrinsic structure of the mathematical entities under consideration, and of their interconnections with other areas of research. He is the type of mathematician that can make tangible and lasting connections with other sciences, esp. physics, because he truly comprehend the science and can thus create coherent and robust mathematical models to explain it.

Over the years we have all enjoyed our interactions with Szafraniec at many international conferences. He is regarded as an individual with utmost wisdom, extremely sharp in his observations, and with a natural ability to focus on what is really important about a scientific matter; in particular, he has a profound understanding of the role of mathematics in science. Szafraniec has established himself as a true expert in topics ranging from moment problems to orthogonal polynomials to unbounded subnormal operator theory to dilation theory, to the theory of Krein spaces, interpolation theory, the quantum harmonic oscillator, canonical commutation relations, and so on. The high level of mathematical excellence so characteristic of Szafraniec's early work has remained present 
throughout his long academic career, representing a clear commitment to quality of research, and making Szafraniec a model for new generations of mathematicians.

\section{Reproducing kernel Hilbert spaces and positive definite kernels}

Functions spaces in which point evaluations are continuous with respect to their norm have been drawing the attention of mathematicians for decades. This feature is enjoyed by the so-called reproducing kernel Hilbert spaces (RKHS) which, together with the positive definite kernels they entail, play an essential role in mathematics and physics. The RKHS theory rooted in the seminal ideas of Zaremba [72] formulated at the beginning of the XX-th century and pushed forward in Aronszajn's work [2] later on.

Suppose that $S$ is a nonempty set, and $(\mathscr{H},\langle\cdot, \cdot\rangle)$ is a Hilbert space of complexvalued functions $f$ on $S$ such that the (Dirac) evaluation map $\delta_{x}: \mathscr{H} \rightarrow \mathbb{C}$ defined by $\delta_{x}(f)=f(x)$ is continuous for all $x \in S$. For every $x \in S$, the Riesz representation theorem applied to $\delta_{x}$ ensures that there is a unique element $K_{x} \in \mathscr{H}$ such that the reproducing kernel property $f(x)=\left\langle f, K_{x}\right\rangle$ holds for all $f \in \mathscr{H}$. In particular, $K_{x}(y)=\left\langle K_{x}, K_{y}\right\rangle$ for all $x, y \in S$. Then the kernel $K: S \times S \rightarrow \mathscr{H}$ defined by $K(x, y)=\left\langle K_{y}, K_{x}\right\rangle$ is a positive definite kernel in the sense that $\sum_{i, j=1}^{n} K\left(x_{i}, x_{j}\right) a_{i} \overline{a_{j}} \geqslant$ 0 for all $n \geqslant 1, x_{1}, \ldots, x_{n} \in S$, and $a_{1}, \ldots, a_{n} \in \mathbb{C}$. The map $K$ is called the reproducing kernel for $\mathscr{H}$. The pair $(\mathscr{H}, K)$ is called a $R K H S$. The MooreAronszajn theorem says that there is a one-to-one correspondence between positive definite kernels and RKHS's.

Szafraniec wrote the monograph [65] in Polish on the general theory of reproducing kernel Hilbert spaces. Among important contributions of Szafraniec to the theory of RKHS and positive definite kernels we may distinguish the following.

Let now $S$ be a $*$-semigroup with unit $e, \mathscr{H}$ be a Hilbert space, $\phi: S \rightarrow \boldsymbol{B}(\mathscr{H})$ be a positive definite map (i.e. $\sum_{i, j=1}^{n}\left\langle\phi\left(s_{j}^{*} s_{i}\right) x_{i}, x_{j}\right\rangle \geqslant 0$ for all $n \geqslant 1, s_{1}, \ldots, s_{n} \in S$, $\left.x_{1}, \ldots, x_{n} \in \mathscr{H}\right)$ and $K_{\phi}: S \times S \rightarrow \boldsymbol{B}(\mathscr{H})$ be defined by $K_{\phi}(t, s)=\phi\left(s^{*} t\right)$. The celebrated Sz.-Nagy general dilation theorem says that if the boundedness condition

$$
\sum_{i, j=1}^{n}\left\langle K_{\phi}\left(u s_{i}, u s_{j}\right) x_{i}, x_{j}\right\rangle \leqslant c(u) \sum_{i, j=1}^{n}\left\langle K_{\phi}\left(s_{i}, s_{j}\right) x_{i}, x_{j}\right\rangle,
$$

holds for all finite sequence $s_{0}, \ldots, s_{n} \in S$ and $x_{0}, \ldots, x_{n} \in \mathscr{H}$ with $c(u)$ independent of the $s_{i}$ and $x_{i}$, then $\phi$ can be represented in the form $\phi(s)=V^{*} \Phi(s) V$, where $V$ is a bounded linear map of $\mathscr{H}$ into a Hilbert space $\mathscr{K}$, and $\Phi$ is a unital $*$-preserving semigroup homomorphism from $S$ into $\boldsymbol{B}(\mathscr{K})$. It was Szafraniec who proved that the Sz.-Nagy general dilation theorem is equivalent to the famous Stinespring dilation theorem for completely positive operator valued linear maps on $C^{*}$-algebras [58]. The following inequality, sometimes called Szafraniec's inequality states that if $\phi$ is positive definite, then for all $u \in S$ and $k \geqslant 1$ (with quantifiers as in (1)), 


$$
\begin{aligned}
\sum_{i, j=1}^{n}\left\langle K_{\phi}\left(u s_{i}, u s_{j}\right) x_{i}, x_{j}\right\rangle \leqslant & \left(\sum_{i, j=1}^{n}\left\langle K_{\phi}\left(s_{i}, s_{j}\right) x_{i}, x_{j}\right\rangle\right)^{1-\frac{1}{2^{k}}} \\
& \times\left(\sum_{i, j=1}^{n}\left\langle K_{\phi}\left(\left(u^{*} u\right)^{2^{k-1}} s_{i},\left(u^{*} u\right)^{2^{k-1}} s_{j}\right) x_{i}, x_{j}\right\rangle\right)^{\frac{1}{2^{k}}} .
\end{aligned}
$$

This inequality enabled Szafraniec to give in [55] a simple proof of the unpublished result due to Arveson saying that if $\phi$ is bounded and positive definite, then $\phi$ satisfies the boundedness condition (1). This idea culminated in proving the following astonishing equivalent version of the boundedness condition (see $[33,56])$ :

$$
\left\langle K_{\phi}(u s, u s) x, x\right\rangle \leqslant c(u)\left\langle K_{\phi}(s, s) x, x\right\rangle, \quad u, s \in S, x \in \mathscr{H},
$$

and led Szafraniec to an extension of the Sz.-Nagy general dilation theorem to the case of unbounded operators by using form approach [61].

An RKHS approach to some holomorphic interpolation problems enabled him to propose a several variable analogue of the classical Pick-Nevanlinna theorem [59], which extends a result of Beatrous and Burbea [6]. This, in turn, was generalized by Quiggin and Barbian [4, 41]. In [64] he applied RKHS and its multiplication operators to model unbounded operators acting on a Hilbert space as in [48] and to investigate $n$-tuples of densely defined operators.

Hilbert $C^{*}$-modules are generalization of Hilbert spaces where the inner products take their values in a $C^{*}$-algebra instead of the field of complex numbers. The theory of positive definite kernels in the setting of Hilbert $C^{*}$-modules was initiated by Murphy [36]. Szafraniec investigated Murphy's result in [69], where he also studied the relation between the notions of complete positivity and positive definiteness for $C^{*}$-algebras; see also [28, 35].

A series of papers [51-54] from the early 70's develops new ideas in the representation theory of function algebras. The earlier results of Mlak and Sarason on decompositions and extensions of uniform algebras were dealing with (mostly contractive) representations in the algebra of bounded Hilbert space operators. The decompositions were obtained with respect to Gleason parts of a uniform algebra. Szafraniec has found a method based on the so called "property R" introduced in his papers [51] and [54] for projections belonging to the dual of Banach space. A special case of this abstract property appears in F. and M. Riesz theorem and in its abstract version on the decomposition of orthogonal measures with respect to Gleason parts of uniform algebras (see [15, II.7]). This allowed to obtain far reaching generalizations, including representations of certain non-commutative algebras on reflexive Banach spaces $X$. In [52] a spectral measure model was constructed for such representations. Another important result was a decomposition for noncontractive representations in a Hilbert space constructed with respect to a family of commuting projections having "property R". For such a decomposition its similarity to the orthogonal decomposition was proved in [54]. 


\section{Decompositions and extensions for operators and relations}

Some twenty years ago the topic of normal extensions of symmetric operators came up. Since this problem is only meaningful in the case where the symmetric operator is not densely defined, it was clear that the context of the problem should be in terms of linear relations in Hilbert spaces. This lead to a number of papers written in collaboration with Hassi, Sebestyén and de Snoo.

The original joint line of research in [23] concerned the decomposition of a linear operator from a Hilbert space $\mathscr{H}$ to a Hilbert space $\mathscr{K}$ into a regular and a singular part, as done by Jørgensen [30] and Ôta [39]. Their ideas formed the right context for the decomposition of any linear relation: if $H$ is a linear relation from $\mathscr{H}$ to $\mathscr{K}$, i.e. a linear subspace of the product Hilbert space $\mathscr{H} \times \mathscr{K}$, then the adjoint relation $H^{*}$ from $\mathscr{K}$ to $\mathscr{H}$ is defined by $H^{*}=J H^{\perp}=(J H)^{\perp}$, where $J$ stands for the flip-flop $J\{f, g\}=\{g,-f\}$ and the orthogonal complement is taken in the appropriate product space. The closure of $H$ is the relation $H^{* *}$ with the multivalued part mul $H^{* *}$ (the linear space of all $g \in \mathscr{K}$ such that $\{0, g\} \in H^{* *}$ ). Let $P$ be the orthogonal projection from $\mathscr{K}$ to mul $H^{* *}$. Then the Lebesgue decomposition of $H$ is given by $H=(I-P) H+P H$, where

$$
(I-P) H=\{\{f,(I-P) g\}:\{f, g\} \in H\}, \quad P H=\{\{f, P g\}:\{f, g\} \in H\}
$$

are the regular part (a closable operator) and the singular part (its closure is a product of closed subspaces). It shares many properties with the corresponding notions from measure theory. The paper [23] was written jointly with Sebestyén, who would continue this line of research with his coworkers in many papers to come.

More results in terms of decompositions of linear relations can be found in an issue of Dissertationes Mathematicae [24]. A relation $H$ is called decomposable if it allows the componentwise sum decomposition $\left.H=H_{0} \widehat{+}(\{0\} \times \operatorname{mul} H)\right\}$, where $H_{0}$ is a closable operator with $\operatorname{ran} H_{0} \perp$ mul $H^{* *}$ and where the componentwise sum is direct. If such an operator exists, then it is automatically equal to the usual orthogonal operator part $H_{\mathrm{op}}$. There is a considerable interplay between the various operator parts and operator-like sum and componentwise sum decompositions. Recall that a linear relation $H$ is called symmetric if $H \subset H^{*}$ and self-adjoint if $H=H^{*}$. The following notions weaken these definitions: a linear relation $H$ is called formally domain-tight if $\operatorname{dom} H \subset \operatorname{dom} H^{*}$ and domain-tight if $\operatorname{dom} H=\operatorname{dom} H^{*}$. The impact of these notions on various componentwise decompositions is studied in detail. In particular, formally domain-tight and domain-tight relations are shown to play an important role in the description of Cartesian decompositions of relations (in a real and an imaginary part).

Extension theory of symmetric operators or relations is to be found in [26]. If $S$ is a bounded symmetric operator in a Hilbert space $\mathscr{H}$, then the self-adjoint extensions $H$ of $S$ can be parameterized as solutions of the completion problem $\left[\begin{array}{cc}S_{11} & S_{21}^{*} \\ S_{21} & *\end{array}\right]$, where the matrix is relative to the orthogonal decomposition $\mathscr{H}=\overline{\operatorname{dom}} S \oplus \operatorname{mul} S^{*}$. By choosing one bounded self-adjoint extension and defining a corresponding 
boundary triplet for $S^{*}$ (see for instance [7]), one may characterize all self-adjoint extensions of $S$, including the unbounded ones. In the case where $S$ is unbounded, there is analogous procedure when $S$ is a maximally nondensely defined operator, defined by the property that $\operatorname{ker}\left(S^{*}-\lambda\right) \cap \overline{\operatorname{dom}} S=\{0\}$ for all $\lambda \in \mathbb{C} \backslash \mathbb{R}$; this condition is equivalent to the requirement that the symmetric relation $S_{\infty}$ defined by $S_{\infty}=S \widehat{+}\left(\{0\} \times\right.$ mul $\left.S^{*}\right)$ be essentially self-adjoint, i.e., $\left(S_{\infty}\right)^{* *}$ be self-adjoint. Note that $S_{\infty}$ is self-adjoint if and only if $\operatorname{dom} S=\overline{\operatorname{dom}} S \cap \operatorname{dom} S^{*}$. Under this condition all self-adjoint extensions of $S$ are in a one-to-one correspondence to the self-adjoint relations $\Theta$ in the Hilbert space mul $S^{*}$ via the perturbation formula $\widetilde{H}+G \Theta G^{*} P$, where $\widetilde{H}$ is a fixed self-adjoint extension of $S$, which is transversal with $S_{\infty}, P$ is the orthogonal projection from $\mathscr{H}$ onto mul $S^{*}$, and $G$ is a boundedly invertible operator from $\mathscr{H}$ onto mul $S^{*}$. Observe that $S_{\infty}$ is the Friedrichs extension of $S$ when $S$ is semibounded.

The topic of extension theory in Hilbert spaces is revisited in [27]. Let $S$ be a symmetric relation in a Hilbert space $\mathscr{H}$. An extension $H$ of $S$ is said to be intermediate if $S \subset H \subset S^{*}$. The self-adjoint extensions of a symmetric relation are by definition all automatically intermediate; they have been described by Coddington [11]. What about normal extensions? First note that a linear relation $H$ in $\mathscr{H}$ is called normal if there exists an isometry $V$ from $H$ onto $H^{*}$ of the form $V\{f, g\}=\{f, h\}$, where $\{f, g\} \in H$ and $\{f, h\} \in H^{*}$. It is known that $H$ is normal if and only if $H$ is closed and $H^{*} H=H H^{*}$. If $S$ is densely defined, then the normal extensions of $S$ are automatically self-adjoint and they are all operators. If $S$ is nondensely defined there may be normal extensions $H$ of $S$ which are not intermediate. Necessary and sufficient conditions for the existence of normal nonself-adjoint intermediate extensions are developed and all such extensions are parameterized. The perturbation formula from [26] plays an important role.

The work [23] with Szafraniec has influenced further work along these lines: it suffices to mention [22], where the original Lebesgue decompositions have been extended to a more general context. Furthermore, the joint work [34] has had a direct influence on [21]. Columns, rows, and blocks are now introduced in [21] not only for operators but also for relations; a simple example of this was already encountered in [26]. Furthermore, Szafraniec was one of the editors of [25] and he turned out to be extremely conscientious and precise in that capacity.

\section{Coherent states}

Professor Szafraniec's interest in coherent states stems from his research concerning basic problems of the operator theory and functional analysis. More than 20 years ago this led him to studies of non-canonical operator structures used to describe generalized models of quantum harmonic oscillator [60, 66], as well as to an extension of his scientific activity to investigation of reproducing kernel Hilbert spaces (RKHS) emerging in quantum physics, in particular coming there from considerations involving coherent states. Mathematical background of the coherent states theory were efforts oriented to problems underlying quantization and unaware using methods of RKHS which motivated Klauder [31] and Bargmann [5] to 
introduce in the early 60' of the previous century harmonic oscillator coherent states (HOCS) as new tools of quantum physics initially treated mere as mathematical objects. Soon after discovered applicability of HOCS in quantum optics gained them high popularity which resulted in more and more extensive investigations of HOCS and their generalizations going beyond those relevant to the standard harmonic oscillator. Two features of coherent states, both standard and generalized, specially attracted Szafraniec's attention. The first was their utility for constructing the SegalBargmann transform as an unitary map which is generated by coherent states and links the Hilbert space of square integrable Schrödinger wave functions $L^{2}(\mathbb{R}, \mathrm{d} x)$ with the Hilbert space $L^{2}(\mathbb{C}, \mu(\mathrm{d} z)) \cap H_{\text {hol }}$ of entire functions while the second was assigning mathematically correct meaning to the so-called resolution of the identity interpreted by the physicists community as sufficient condition enabling them to use coherent states as overcomplete non-orthogonal reference frames. Szafraniec soon recognized that the right tool to analyze and understand both problems is to apply the RKHS methods and become one of the forerunners of such methodology $[62,65]$.

The novel feature of Szafraniec's approach was to use his author's formalism of the RKHS theory. The crucial elements which enable to apply this formalism are: i.) to introduce a set of complex valued functions $\left\{\Phi_{n}(x)\right\}_{n}$ of $x \in X$ which satisfy $\sum_{n} \bar{\Phi}_{n}(x) \Phi_{n}(x)<\infty$ for all $x$ (if it happens, $\Phi_{n}(x)$ 's are called the kernel functions) and ii) to construct the reproducing kernel according to $K(x, y)=\sum_{n} \Phi_{n}(x) \bar{\Phi}_{n}(y)$. Having the set $\left\{\Phi_{n}(x)\right\}_{n}$ one may proceed further and construct prospective generalized coherent states $c_{x}=\sum_{n} \Phi_{n}(x) e_{n}$ with the Hilbert space basis $\left\{e_{n}\right\}_{n}$ entering the game. In the Section "Komentarze" ("Comments") closing the Chapter 3 of his book [65] entitled Reproducing kernel Hilbert spaces (unfortunately available only in the Polish edition published by the Jagiellonian University) Szafraniec emphasized the validity of the RKHS structure for the construction of the Segal-Bargmann space and wrote "It should be noted here that kernel functions in the Segal-Bargmann space are an example of coherent states, the notion appearing in the quantum mechanics". The RKHS approach to the theory of generalized coherent states, being during last ten years developed by Szafraniec in collaboration with Ali, Górska and Horzela, provided interesting results. Here should be mentioned a new look at the resolution of the identity understood in the context of the Segal-Bargmann transform [29] and explicit construction of the single particle and bipartite Hermite coherent states built with holomorphic Hermite polynomials of single and two variables used to construct suitable kernel functions [13, 20]. Such formed coherent states obey physically interesting properties-for single variable case they are the squeezed states of quantum optics [1] while for two variable case represent quantum states which minimize the Heisenberg uncertainty relations, i.e. are squeezed-coherent, and (what is astonishing) at the same time entangled, i.e. non-factorizable, which signalizes coexistence of coherence and non-dynamical correlations [19].

Another problem of the coherent states theory studied by Szafraniec (together with Górska and Horzela) was inconsistency of naive generalization of squeezed states disturbing physicists for many years [14]. The operator $S_{k}(\xi)=\exp \left(\xi a^{\dagger k}-\right.$ 
$\left.\bar{\xi} a^{k}\right)$ used to define HOCS for $k=1$ and the squeezed states for $k=2$ becomes meaningless if $k \geq 3$ as its matrix elements are given by divergent series. This happens although the operator in the exponent remains antihermitean which many physicists are used to treat as implying the unitarity of $S$. Szafraniec and his collaborators explained this puzzle showing that the operator $\xi a^{\dagger k}-\bar{\xi} a^{k}$ has deficiency indices equal to 0 as long as $k=1,2$ and thus it is self-adjoint. But for $k>2$ its deficiency indices are equal to $(k, k)$ so it is only essentially self-adjoint and $S_{k}(\xi)$ is not unitary [18]. If lacks unitarity it does not fit to the standard quantum mechanical formalism and should not be used in any routine way.

\section{Quantization}

Theoretical physicists do not give the same constraints as would do a mathematician when writing an article. This means that physicist's approach to problems is often more intuitive than mathematically demonstrative. One could find in Szafraniec a very open-minded and helpful character, able to seize fully physicist's intuition while fully respecting mathematical rigor. One could learn a lot from Szafraniec when considering problems pertaining to the formalism of Quantum Physics. Szafraniec fully understands subtleties of quantum formalism and is able to share this understanding with a large community of physicists. On the other hand, he is a pure mathematician, with outstanding expertise in unbounded operator theory, moment problems, orthogonal polynomials, and in rigorous formulation of the essence of Quantum Mechanics, namely the canonical commutation rule. To be more precise here, the major mathematical contribution of Szafraniec on the latter question is a characterization, in terms of subnormality, of the canonical solution (creation-annihilation) of the commutation relation of the quantum harmonic oscillator.

Since the advent of quantum mechanics one recurrent question concerns the transition from classical to quantum models (i.e., quantization) for some system, regardless its physical relevance. One interesting method is to use generalized coherent states and more generally positive operator valued measure (POVM) to implement what is named integral quantization. To some extent, the latter is similar to the Berezin-Toeplitz quantization. In a nutshell, the Berezin-Toeplitz quantization of a symplectic manifold $M$ with Kähler structure maps functions on $M$ to operators in the Hilbert space of square-integrable holomorphic sections of an appropriate complex line bundle. Denoting by $\Pi$ the orthogonal projection operator from the space of all square-integrable sections to the holomorphic subspace, for any bounded measurable function $f$, one constructs the Toeplitz operator $A_{f}$ with symbol $f$, acting on the space of holomorphic sections, as $A_{f} \phi=\Pi(f \phi)$. That is, $A_{f} \phi$ consists of multiplication by $f$ followed by projection back into the holomorphic subspace. Two Szafraniec's papers, $[62,63]$ were at the origin of two articles devoted to this integral quantization with coherent states. In the first one, [16], a coherent state quantization of the complex plane was presented when the latter is equipped with a non rotationally invariant measure. While the canonical commutation rule (up to a simple rescaling) still holds true, these authors explained 
how the involved coherent states, built from holomorphic continuations of Hermite polynomials, are related to the non-commutative plane. In the second paper, [17], they examined mathematical questions around angle (or phase) operator associated with a number operator through a short list of basic requirements, and they have implemented three methods of construction of quantum angle. The first one is based on operator theory and parallels the definition of angle for the upper half-circle through its cosine and completed by a sign inversion. The two other methods are based on the integral quantization with adapted coherent states.

Now, a basic requirement in the construction of coherent states is the resolution of identity, which usually invokes an appropriate measure. In the process of generalization of coherent states, it may be advantageous to have a construction which does not explicitly make use of a measure. As a matter of fact, a measure-free construction was developed in other Szafraniec's papers, like [29]. The key point is the existence of a sequence of complex functions satisfying a certain convergence criterion. The reproducing kernel Hilbert space, required for the coherent states, can be constructed out of these functions. Examples are provided where these sequences appear, e.g. in moment problems and orthogonal polynomials.

Open Access This article is licensed under a Creative Commons Attribution 4.0 International License, which permits use, sharing, adaptation, distribution and reproduction in any medium or format, as long as you give appropriate credit to the original author(s) and the source, provide a link to the Creative Commons licence, and indicate if changes were made. The images or other third party material in this article are included in the article's Creative Commons licence, unless indicated otherwise in a credit line to the material. If material is not included in the article's Creative Commons licence and your intended use is not permitted by statutory regulation or exceeds the permitted use, you will need to obtain permission directly from the copyright holder. To view a copy of this licence, visit http:// creativecommons.org/licenses/by/4.0/.

\section{References}

1. Ali, S.T., Górska, K., Horzela, A., Szafraniec, F.H.: Squeezed states and Hermite polynomials in a complex variable. J. Math. Phys. 55(1), 012107 (2014)

2. Aronszajn, N.: Theory of reproducing kernels. Trans. Am. Math. Soc. 68, 337-404 (1950)

3. Bade, W.G.: Unbounded spectral operators. Pac. J. Math. 4, 373-392 (1954)

4. Barbian, C.: A characterization of multiplication operators on reproducing kernel Hilbert spaces. J. Oper. Theory 65, 235-240 (2011)

5. Bargmann, V.: On a Hilbert space of analytic functions and an associated integral transform. Commun. Pure Appl. Math. 14, 187-214 (1961)

6. Beatrous, F., Burbea, J.: Positive-definiteness and its applications to interpolation problems for holomorphic functions. Trans. Am. Math. Soc. 284, 247-270 (1984)

7. Behrndt, J., Hassi, S., de Snoo, H.S.V.: Boundary Value Problems, Weyl Functions, and Differential Operators. Monographs in Mathematics vol. 108, Birkhäuser (2020)

8. Cichoń, D., Littlejohn, L., Stochel, J.: Franciszek Hugon Szafraniec: a scholar of eminence. Complex Anal. Oper. Theory 6, 529-531 (2012)

9. Cichoń, D., Stochel, J., Szafraniec, F.: The complex moment problem: determinacy and extendibility. Math. Scand. 124, 263-288 (2019)

10. Coddington, E.A.: Formal normal operators having no normal extension. Can. J. Math. 17, 1030-1040 (1965)

11. Coddington, E.A.: Extension Theory of Formally Normal and Symmetric Subspaces. Mem. Am. Math. Soc. 134 (1973) 
12. Conway, J.B.: The Theory of Subnormal Operators. Mathematical Surveys and Monographs, 36. American Mathematical Society, Providence, RI (1991)

13. van Eijndhoven, S.J.L., Meyers, J.L.H.: New orthogonality relations for the Hermite polynomials and related Hilbert spaces. J. Math. Anal. Appl. 146, 89-98 (1990)

14. Fisher, R.A., Nieto, M.M., Sandberg, V.D.: Impossibility of naively generalizing squeezed coherent states. Phys. Rev. D 29, 1107-1110 (1984)

15. Gamelin, T.W.: Uniform Algebras. Prentice Hall Inc, Englewood Clifs, NJ (1969)

16. Gazeau, J.-P., Szafraniec, F.H.: Holomorphic Hermite polynomials and a non-commutative plane. J. Phys. A 44, 495201 (2011). 13 pp

17. Gazeau, J.-P., Szafraniec, F.H.: Three paths toward the quantum angle operator. Ann. Phys. 375, $16-35$ (2016)

18. Górska, K., Horzela, A., Szafraniec, F.H.: Squeezing: the ups and downs. Proc. R. Soc. A 470, 20140205 (2014)

19. Górska, K., Horzela, A., Szafraniec, F.H.: Coherence, squeezing and entanglement - an example of peaceful coexistence, Ch. 5, pp. 89-117, in Coherent states and their applications: A contemporary panorama (Springer, Berlin, 2018)

20. Górska, K., Horzela, A., Szafraniec, F.H.: Holomorphic Hermite polynomials in two variables. J. Math. Anal. Appl. 470, 750-772 (2019)

21. Hassi, S., Labrousse, J.-Ph., de Snoo, H.S.V.: Operational calculus for rows, columns, and blocks of linear relations. Adv. Oper. Theory (to appear)

22. Hassi, S., Sebestyén, Z., de Snoo, H.S.V.: Lebesgue type decompositions for linear relations and Ando's uniqueness criterion. Acta Sci. Math. (Szeged) 84, 465-507 (2018)

23. Hassi, S., Sebestyén, Z., de Snoo, H.S.V., Szafraniec, F.H.: A canonical decomposition for linear operators and linear relations. Acta Math. Hungar. 115, 281-307 (2007)

24. Hassi, S., de Snoo, H.S.V., Szafraniec, F.H.: Componentwise and canonical decompositions of linear relations. Dissertationes Mathematicae 465 (2009)

25. Hassi, S., de Snoo, H.S.V., Szafraniec, F.H. (editors): Operator methods for boundary value problems. Lecture Notes 404, London Mathematical Society (2012)

26. Hassi, S., de Snoo, H.S.V., Szafraniec, F.H.: Infinite-dimensional perturbations, maximally nondensely defined symmetric operators, and some matrix representations. Indag. Math. 23, 1087-1117 (2012)

27. Hassi, S., de Snoo, H.S.V., Szafraniec, F.H.: Normal intermediate extensions of symmetric relations. Acta Math. Szeged 80, 195-232 (2014)

28. Heo, J.: Reproducing kernel Hilbert $C^{*}$-modules and kernels associated with cocycles. J. Math. Phys. 49, 103507 (2008). $12 \mathrm{pp}$

29. Horzela, A., Szafraniec, F.H.: A measure-free approach to coherent states. J. Phys. A 45, 244018 (2012). 9 pp

30. Jørgensen, P.E.T.: Unbounded operators: perturbations and commutativity problems. J. Funct. Anal. 39, 281-307 (1980)

31. Klauder, J.R.: The action option and a Feynman quantization of spinor fields in terms of ordinary c-numbers. Ann. Phys. 11, 123-168 (1960)

32. Landau, H.J.: Classical background of the moment problem, in Moments in Mathematics. Proceeding of Symposia Application Mathematics, 37, AMS Short Course Lecture Notes, American Mathematics Society, Providence, RI, pp. 1-15 (1987)

33. Masani, P.: Dilations as propagators of hilbertian varieties. SIAM J. Math. Anal. 9, 414-456 (1978)

34. Möller, M., Szafraniec, F.H.: Adjoints and formal adjoints of matrices of unbounded operators. Proc. Am. Math. Soc. 136, 2165-2176 (2008)

35. Moslehian, M.S.: Conditionally positive definite kernels in Hilbert $C^{*}$-modules. Positivity 21, 1161-1172 (2017)

36. Murphy, G.J.: Positive definite kernels and Hilbert $C^{*}$-modules. Proc. Edinburgh Math. Soc. (2) 40, 367-374 (1997)

37. Naimark, M.A.: Spectral functions of a symmetric operator. Izv. Akad. Nauk 7, 285-296 (1943)

38. Nussbaum, A.E.: Quasi-analytic vectors. Ark. Mat. 6, 179-191 (1971)

39. Ôta, S.: On a singular part of an unbounded operator. Z. Anal. Anwendungen 7, 15-18 (1988)

40. Putinar, M., Vasilescu, F.-H.: Solving moment problems by dimensional extension. Ann. Math. 149, 1087-1107 (1999)

41. Quiggin, P.: For which reproducing kernel Hilbert spaces is Pick's theorem true? Integr. Equ. Oper. Theory 16, 244-266 (1993) 
42. Schmüdgen, K.: A formally normal operator without normal extension. Proc. Am. Math. Soc. 98, 503-504 (1985)

43. Schmüdgen, K.: The $K$-moment problem for compact semi-algebraic sets. Math. Ann. 289, 203-206 (1991)

44. Stochel, J.: Moment functions on real algebraic sets. Ark. Mat. 30, 133-148 (1992)

45. Stochel, J., Szafraniec, F.H.: A characterization of subnormal operators, Spectral theory of linear operators and related topics (Timi soara/Herculane, 1983), 261-263, Oper. Theory Adv. Appl., 14, Birkhäuser, Basel (1984)

46. Stochel, J., Szafraniec, F.H.: On normal extensions of unbounded operators. I. J. Oper. Theory 14, 31-55 (1984)

47. Stochel, J., Szafraniec, F.H.: On normal extensions of unbounded operators. II. Acta Sci. Math. (Szeged) 53, 153-177 (1989)

48. Stochel, J., Szafraniec, F.H.: On normal extensions of unbounded operators. III. Publ. RIMS Kyoto Univ. J. 25, 105-139 (1989)

49. Stochel, J., Szafraniec, F.: Algebraic operators and moments on algebraic sets. Portugal. Math 51, 25-45 (1994)

50. Stochel, J., Szafraniec, F.H.: The complex moment problem and subnormality: a polar decomposition approach. J. Funct. Anal. 159, 432-491 (1998)

51. Szafraniec, F.H.: Decomposition of operator valued representations of Banach algebras. Bull. Acad. Polon. Sci. Sér. Sci. Math. Astron. Phys. 18, 321-324 (1970)

52. Szafraniec, F.H.: Some spectral properties of operator-valued representations of function algebras. Ann. Polon. Math. 25, 187-194 (1971)

53. Szafraniec, F.H.: Orthogonal decompositions of non-contractive operator valued representations of Banach algebras. Bull. Acad. Polon. Sci., Sér. Sci. Math. Astron. Phys. 19, 937-940 (1971)

54. Szafraniec, F.H.: Decompositions of non-contractive operator-valued representations of Banach algebras. Stud. Math. 42, 97-108 (1972)

55. Szafraniec, F.H.: On the boundedness condition involved in a general dilation theory. Bull. Acad. Polon. Sci. Ser. Sci. Math. Astron. Phys. 24, 877-881 (1976)

56. Szafraniec, F.H.: A propos of professor Masani's talk. In: Probability theory on vector spaces, Proceedings, Trzebieszowice, Poland 1977 (Ed.: A. Woron). Lecture Notes in Mathematics 656, Springer, Berlin, pp. 245-249 (1978)

57. Szafraniec, F.H.: Boundedness of the shift operator related to positive definite forms: an application to moment problems. Ark. Mat. 19, 251-259 (1981)

58. Szafraniec, F.H.: Dilations of linear and nonlinear maps, in Functions, Series, Operators, Proceedings, Budapest (Hungary), 1980, Sz.-Nagy, B., Szabados, J. (eds.), Colloquia Mathematica Societatis János Bolyai, vol. 35, pp. 1165-1169, North-Holland, Amsterdam (1983)

59. Szafraniec, F.H.: On bounded holomorphic interpolation in several variables. Monatsh. Math. 101, 59-66 (1986)

60. Szafraniec, F.H.: A RKHS of entire functions and its multiplication operator. An explicit example, in Linear Operators in Function Spaces, Proceedings, Timişoara (Romania), June 6-16, 1988. Helson, H., Sz. Nagy, B., Vasilescu, F.-H. (eds.) Operator Theory: Advances and Applications, vol. 43, pp. 309-312, Birkhäuser, Basel (1990)

61. Szafraniec, F.H.: The Sz.-Nagy "théorème principal" extended. Application to subnormality. Acta Sci. Math. (Szeged) 57, 249-262 (1993)

62. Szafraniec, F.H.: Analytic models of the quantum harmonic oscillator. Contemp. Math. 212, 269-276 (1998)

63. Szafraniec, F.H.: Subnormality in the quantum harmonic oscillator. Commun. Math. Phys. 210, 323-334 (2000)

64. Szafraniec, F.H.: The reproducing kernel Hilbert space and its multiplication operators. In: Complex analysis and related topics (Cuernavaca, 1996), 253-263, Oper. Theory Adv. Appl., 114, Birkhäuser, Basel (2000)

65. Szafraniec, F.H.: Przestrzenie Hilberta z jądrem reprodukującym (Reproducing kernel Hilbert spaces), in Polish, Wydawnictwo Uniwersytetu Jagiellońskiego, Kraków (2004)

66. Szafraniec, F.H.: Operators of the $q$-oscillator. Banach Center Publ. 78, 293-307 (2007)

67. Szafraniec, F.H.: How to recognize the creation operator. Rep. Math. Phys. 59, 401-408 (2007)

68. Szafraniec, F.H.: Normals, subnormals and an open question. Oper. Matrix. 4, 485-510 (2010) 
69. Szafraniec, F.H.: Murphy's Positive definite kernels and Hilbert $C^{*}$-modules reorganized. In: Noncommutative harmonic analysis with applications to probability II, 275-295, Banach Center Publ. 89, Polish Acad. Sci. Inst. Math., Warsaw (2010)

70. Szafraniec, F., Wojtylak, M.: The Sobolev moment problem and Jordan dilations. J. Math. Anal. Appl. 444, 1675-1689 (2016)

71. Szafraniec, F., Wojtylak, M.: The complex moment problem of Dirichlet type, submitted (2020)

72. Zaremba, S.: L'équation biharmonique et une classe remarquable de fonctions fondamentales harmoniques. Bulletin International de l'Académie des Sciences de Cracovie 147-196 (1907)

\section{Affiliations}

\section{Raúl E. Curto ${ }^{1}$ - Jean-Pierre Gazeau ${ }^{2} \cdot$ Andrzej Horzela $^{3}$. Mohammad Sal Moslehian ${ }^{4} \cdot$ Mihai Putinar $^{5,6} \cdot$ Konrad Schmüdgen $^{7}$. Henk de Snoo $^{8} \cdot$ Jan Stochel ${ }^{9}$}

Raúl E. Curto

raul-curto@uiowa.edu

Jean-Pierre Gazeau

gazeau@apc.in2p3.fr

Andrzej Horzela

Andrzej.Horzela@ifj.edu.pl

Mohammad Sal Moslehian

moslehian@um.ac.ir; moslehian@yahoo.com

Mihai Putinar

mputinar@math.ucsb.edu; mihai.putinar@ncl.ac.uk

Konrad Schmüdgen

schmuedgen@math.uni-leipzig.de

Henk de Snoo

hsvdesnoo@gmail.com

1 The University of Iowa, Iowa City, IA 52242, USA

2 APC (UMR 7164), Department of Physics, Université Paris-Diderot, 75205 Paris, France

3 Department of Mathematical Physics, H. Niewodniczański Institute of Nuclear Physics, Polish Academy of Sciences, ul. Eljasza-Radzikowskiego 152, 31342 Kraków, Poland

4 Department of Pure Mathematics, Ferdowsi University of Mashhad, Center of Excellence in Analysis on Algebraic Structures (CEAAS), P.O. Box 1159, Mashhad 91775, Iran

5 University of California at Santa Barbara, Santa Barbara, CA, USA

6 Newcastle University, Newcastle Upon Tyne, UK

7 Mathematical Institute, University of Leipzig, Augustusplatz 10, 04109 Leipzig, Germany

8 Bernoulii Institute for Mathematics, Computer Science and Artificial Intelligence, University of Groningen, P.O. Box 407, 9700 AK Groningen, The Netherlands

9 Instytut Matematyki, Uniwersytet Jagielloński, ul. Łojasiewicza 6, PL 30348 Kraków, Poland 